\title{
Discordance of Non-HDL and Directly Measured LDL Cholesterol: Which Lipid Measure is Preferred When Calculated LDL Is Inaccurate?
}

\author{
Lawrence Baruch, ${ }^{1,2}$ Valerie J. Chiong, ${ }^{1}$ Sanjay Agarwal, ${ }^{3}$ and Bhanu Gupta ${ }^{4}$ \\ ${ }^{1}$ James J. Peters VA Medical Center, Bronx, NY 10468, USA \\ ${ }^{2} \mathrm{Mt}$. Sinai School of Medicine, NY, USA \\ ${ }^{3}$ Confluence Health, Moses Lake, WA 98837, USA \\ ${ }^{4}$ St. Luke's Hospital, University of Missouri, Kansas City, MO 64111, USA \\ Correspondence should be addressed to Lawrence Baruch; lawrence.baruch@va.gov
}

Received 12 February 2013; Accepted 25 March 2013

Academic Editor: Gerhard M. Kostner

Copyright (C) 2013 Lawrence Baruch et al. This is an open access article distributed under the Creative Commons Attribution License, which permits unrestricted use, distribution, and reproduction in any medium, provided the original work is properly cited.

\begin{abstract}
Objective. To determine if non-HDL cholesterol (N-HDL) and directly measured LDL cholesterol (D-LDL) are clinically equivalent measurements. Patients and Methods. Eighty-one subjects recruited for 2 cholesterol treatment studies had at least 1 complete fasting lipid panel and D-LDL performed simultaneously; 64 had a second assessment after 4 to 6 weeks, resulting in 145 triads of C-LDL, D-LDL, and N-HDL. To directly compare N-HDL to D-LDL and C-LDL, we normalized the N-HDL by subtracting 30 from the $\mathrm{N}-\mathrm{HDL}\left(\mathrm{N}-\mathrm{HDL}_{\mathrm{A}}\right)$. Results. There was significant correlation between N-HDL $\mathrm{A}_{\mathrm{A}}$, D-LDL, and C-LDL. Correlation was significantly greater between $\mathrm{N}-\mathrm{HDL}_{\mathrm{A}}$ and C-LDL than between $\mathrm{N}-\mathrm{HDL}_{\mathrm{A}}$ and D-LDL. A greater than $20 \mathrm{mg} / \mathrm{dL}$ difference between measures was observed more commonly between N-HDL $\mathrm{A}_{\mathrm{A}}$ and D-LDL, 29\%, than between C-LDL and N-HDL $\mathrm{A}_{\mathrm{A}}, 11 \%(P<0.001)$, and CLDL and D-LDL, 17\% $(P=0.028)$. Clinical discordance was most common, and concordance was least common between N-HDL and D-LDL. Conclusions. Our findings suggest that N-HDL cholesterol and D-LDL cholesterol are not clinically equivalent and frequently discordant. As N-HDL may be superior to even C-LDL for predicting events in statin-treated patients, utilizing N-HDL to guide therapy would appear to be preferable to D-LDL when C-LDL is inaccurate.
\end{abstract}

\section{Introduction}

Calculated low density lipoprotein cholesterol (LDL) is the cornerstone of lipid lowering therapy [1-3]. In certain clinical situations, namely, the fed state or when triglycerides are greater than $400 \mathrm{mg} / \mathrm{dL}$, calculated LDL is inaccurate and the guidelines recommend the use of directly measured LDL cholesterol [1].

We previously reported that calculated and directly measured LDL are not clinically equivalent when targeting ATP III goals with lipid lowering therapy in a significant number of patients [4]. Based on this finding, we recommended that non-HDL cholesterol (N-HDL), an accepted, inexpensive, and guideline-based measure of atherogenic particles, available from the routine lipid panel, replace directly measured LDL (D-LDL) when the calculated LDL (C-LDL) is inaccurate [4]. However, few studies are available comparing the clinical equivalence of calculated LDL, directly measured LDL, and non-HDL cholesterol measurements. Therefore, we evaluated the clinical equivalence of $\mathrm{N}-\mathrm{HDL}$ and D-LDL which would allow us to determine which lipid measure is more appropriate when C-LDL is inaccurate.

\section{Methods}

Our population consisted of 81 subjects from the Bronx Veterans Affairs Medical Center recruited for 2 research studies from January 2007 through March 2009. The studies incorporated simultaneous measurement of direct LDL with a Siemens Advia Chemistry System and a complete fasting lipid panel at 2 time intervals, baseline, and after 4 to 6 
weeks of therapy. C-LDL was calculated using the Friedwald formula: total cholesterol-HDL - triglycerides/5. Non-HDL cholesterol was calculated as total cholesterol-HDL cholesterol. The studies were approved by the institutional review board of the Bronx VA Medical Center and registered at http://clinicaltrials.gov/ (NCT00762164 and NCT00762229). All subjects signed an informed consent document.

The NCEP ATP III guidelines, which established that NHDL goals are $30 \mathrm{mg} / \mathrm{dL}$ above those for LDL (e.g., an individual with an LDL goal $<100$ has an N-HDL goal of $<130$ ), formed the basis for our comparisons between the measures of LDL (direct and calculated) and N-HDL $[1,3]$. Thus, for assessment of placement in the same lipid goal cut points, LDL goals of $<160,<130,<100$, and $<70 \mathrm{mg} / \mathrm{dL}$ corresponded to N-HDL goals of $<190,<160,<130$, and $<100 \mathrm{mg} / \mathrm{dL}$, respectively $[1,3]$. To "normalize" N-HDL to LDL values for direct numerical comparisons, 30 was subtracted from $\mathrm{N}$ HDL (e.g., an N-HDL of 155 was "normalized" to a value of $125,155-30=125)$ to create the "adjusted" N-HDL (N$\mathrm{HDL}_{\mathrm{A}}$ ).

Correlation between the lipid parameters was determined using Pearson's correlation coefficient.

The relationship between C-LDL, D-LDL, and N-HDL was also evaluated from a clinical perspective based on the following rationale. In clinical practice, adjustment of lipid lowering therapy is primarily based on the LDL numerical value (e.g., $112 \mathrm{mg} / \mathrm{dL}$ ) and the ATP III goal (e.g., LDL < $100 \mathrm{mg} / \mathrm{dL}, \mathrm{N}-\mathrm{HDL}<130 \mathrm{mg} / \mathrm{dL}$ in a patient with diabetes) $[1,3]$. Furthermore, doubling the dose of any statin results in an additional 6\% reduction in LDL [5]. Finally, minor changes in LDL are not generally considered to be clinically meaningful. As such, when comparing any 2 of the lipid values (e.g., N-HDL to D-LDL, N-HDL to C-LDL) they were considered "clinically concordant" when fulfilling the following criteria: (1) placement in the same ATP III goal cut point range (e.g., 100-129 when comparing C-LDL and D-LDL, an N-HDL of 100-129 and a C-LDL 70-99) [1,3], (2) $<6 \%$ difference between the values (e.g., C-LDL versus $\mathrm{N}-\mathrm{HDL}_{\mathrm{A}}$, and C-LDL versus D-LDL) which represents the incremental LDL lowering provided by a single titration of statin dose (e.g., from simvastatin 20 to $40 \mathrm{mg}$ [5]), and (3) $<10 \mathrm{mg} / \mathrm{dL}$ difference between the 2 values (a difference that clinicians may consider meaningful, thus not concordant, even when the values being compared are in the same ATP III cut point and differ by $<6 \%$, e.g., when D-LDL is $181 \mathrm{mg} / \mathrm{dL}$ and $\mathrm{N}-\mathrm{HDL}_{\mathrm{A}}$ is $171 \mathrm{mg} / \mathrm{dL}$ ).

We considered the numerical values "clinically discordant" when they fulfilled the following criteria: (1) placement in different ATP III goal cut point ranges [1, 3], $(2) \geq 12 \%$ difference between the 2 values (representing the incremental LDL lowering provided by two statin titration steps, e.g., from atorvastatin 20 to $80 \mathrm{mg}$ [5]), and (3) $\geq 10 \mathrm{mg} / \mathrm{dL}$ difference between the values (as clinicians may not consider the values discordant, even when they are in different ATP III cut points and differ by $\geq 12 \%$ when the values being compared differ by $<10 \mathrm{mg} / \mathrm{dL}$, e.g., when $\mathrm{N}-\mathrm{HDL}_{\mathrm{A}}$ is $66 \mathrm{mg} / \mathrm{dL}$ and $\mathrm{D}-\mathrm{LDL}$ is $75 \mathrm{mg} / \mathrm{dL}$ ).

Categorical data were compared using descriptive statistics from Minitab 15th Edition (State College, PA). One
TABLE 1: Baseline characteristics.

\begin{tabular}{|c|c|}
\hline Variable & Initiation $(n=81)$ \\
\hline Age (years) & 60 \\
\hline Weight $(\mathrm{kg})^{*}$ & 92 \\
\hline Height $(\mathrm{cm})^{*}$ & 176 \\
\hline Male & $38(100 \%)$ \\
\hline Hypertension & $25(66 \%)$ \\
\hline Diabetes mellitus & $9(24 \%)$ \\
\hline Low density lipoprotein $(\mathrm{mg} / \mathrm{dL})$ - calculated ${ }^{*}$ & 141 \\
\hline Low density lipoprotein $(\mathrm{mg} / \mathrm{dL})$ - direct ${ }^{*}$ & 149 \\
\hline Total cholesterol (mg/dL) & 216 \\
\hline $\mathrm{HDL}(\mathrm{mg} / \mathrm{dL})$ & 49 \\
\hline Non-HDL (mg/dL) & 160 \\
\hline "Adjusted" non-HDL (mg/dL) & 130 \\
\hline Triglycerides $(\mathrm{mg} / \mathrm{dL})$ & 130 \\
\hline
\end{tabular}

*One patient had nonfasting lipids; his baseline LDL measurements, triglycerides, HDL, and "adjusted" non-HDL were therefore not included. Two patients did not have an assessment of height and weight.

sample $t$-test and analysis of variance (ANOVA) were used to assess within-group and between-group differences in lipid values. A $P$ value $<0.05$ was considered statistically significant for these analyses. Differences in correlation coefficients were evaluated by Fisher's $z$-transformation. A $z$-value $\geq 2.0$ was considered significant for these analyses. Microsoft Excel 2007 and SPSS, version 19.0 (SPSS Inc., an IBM company), were used to analyze study data.

Our sample size was limited by the number of subjects who enrolled in each of the respective protocols.

\section{Results}

A total of 81 subjects had at least one lipid triad of C-LDL, D-LDL, and N-HDL performed simultaneously. Of these 81 subjects, 64 successfully completed their respective studies and had lipids assessed at baseline and followup. Sixteen subjects had lipids assessed only at baseline, and 1 patient only had a followup, as his baseline lipid assessment was nonfasting. This resulted in a total of 145 total triads of C-LDL, D-LDL, and N-HDL that were included in the analysis.

Baseline characteristics are shown in Table 1. Subjects tended to be elderly, overweight, hypertensive, and predominantly male. At baseline, $\mathrm{N}-\mathrm{HDL}_{\mathrm{A}}$ was less than direct and calculated LDL in $81 \%$ and $68 \%$ of subjects, respectively; DLDL was greater than C-LDL in $66 \%$ of patients.

There was significant correlation between $\mathrm{N}-\mathrm{HDL}_{\mathrm{A}}, \mathrm{D}-$ LDL, and C-LDL. For the entire cohort, which included all 145 baseline and follow-up values, the correlations for $\mathrm{N}$ $\mathrm{HDL}_{\mathrm{A}}$ with C-LDL, $r^{2}$ of 0.89 (Figure $1(\mathrm{a})$ ), N-HDL $\mathrm{A}_{\mathrm{A}}$ with D-LDL, $r^{2}$ of 0.80 (Figure 1(b)), and C-LDL with D-LDL, $r^{2}$ of 0.86 (Figure $1(\mathrm{c})$ ) were strong. The strength of the correlation between $\mathrm{N}-\mathrm{HDL}_{\mathrm{A}}$ and C-LDL was statistically significantly greater than between $\mathrm{N}-\mathrm{HDL}_{\mathrm{A}}$ and $\mathrm{D}$-LDL for both the baseline $(z=2.02)$ and entire cohorts $(z=2.66)$.

At baseline, clinical concordance (as defined in Section 2) between D-LDL and N-HDL was present in only $20 \%$ 


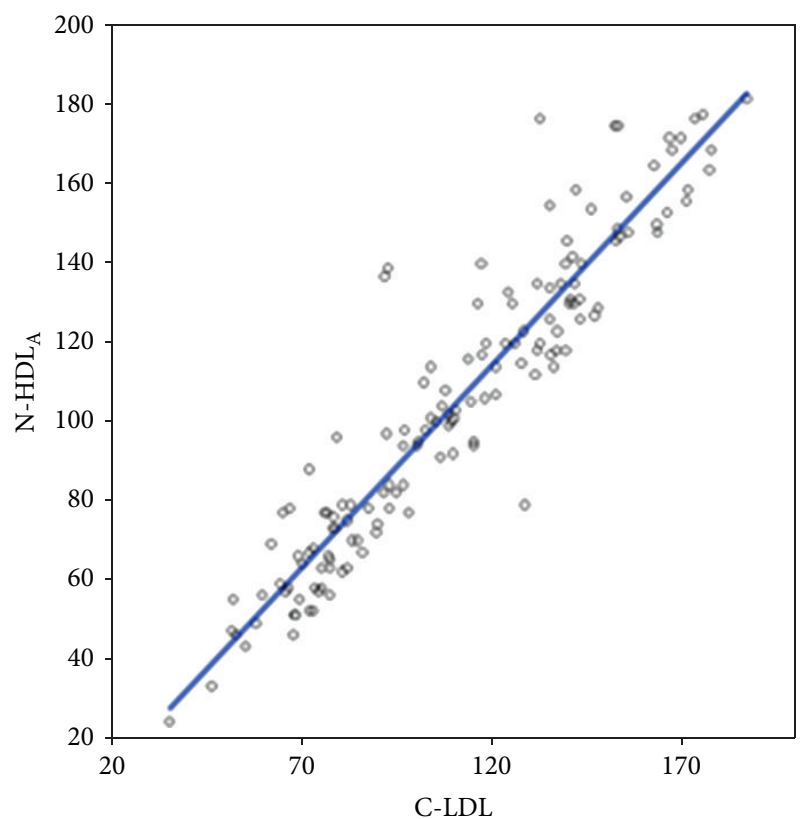

(a)

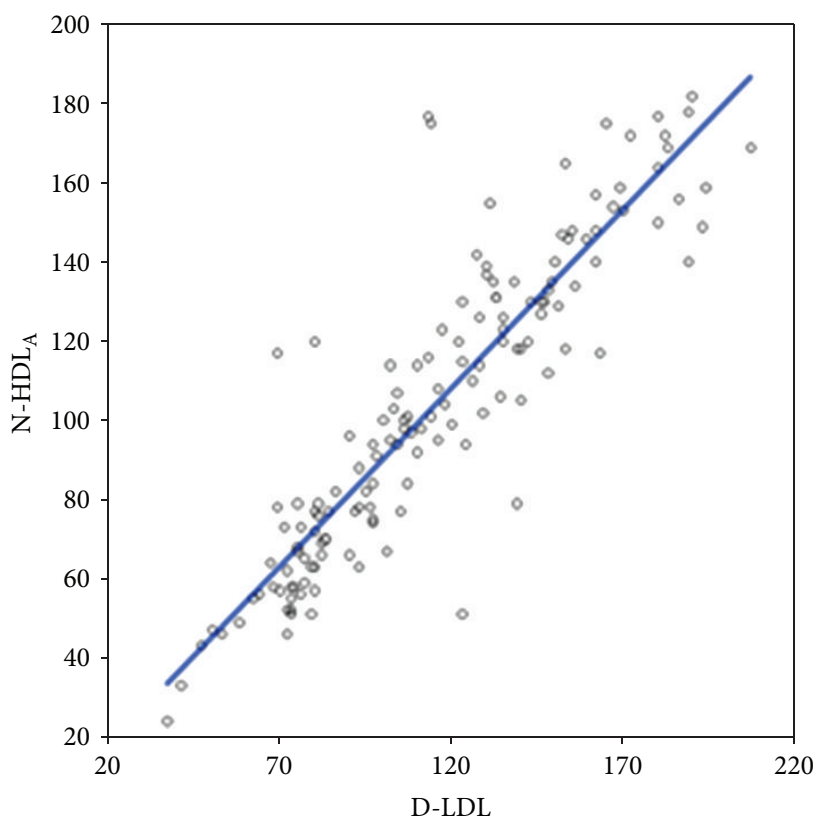

(b)

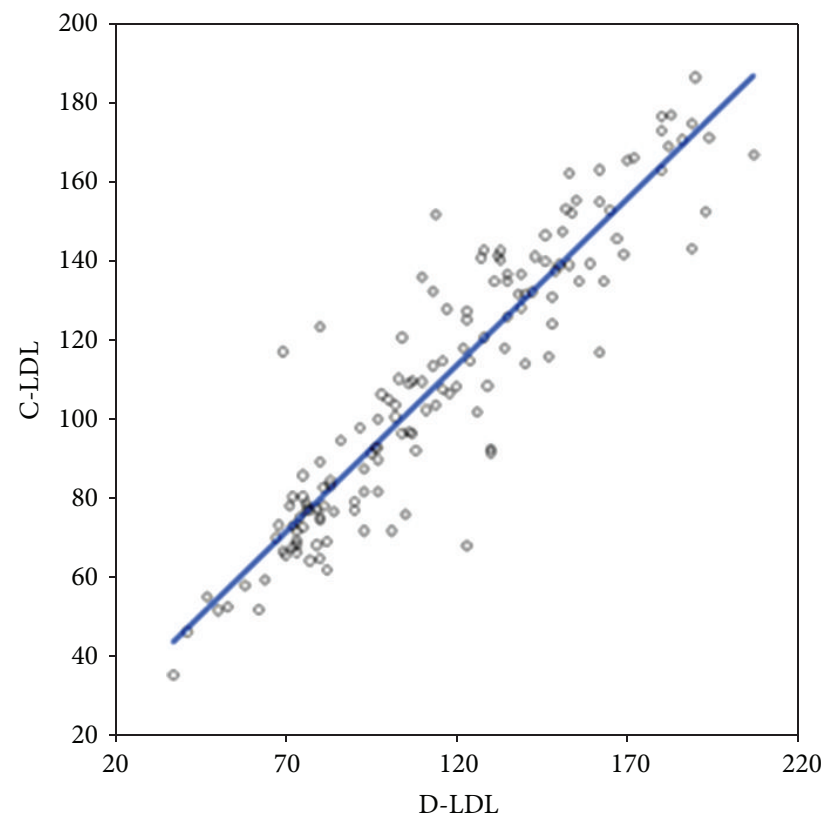

(c)

Figure 1: Regression plots for D-LDL-C, C-LDL, and N-HDL $\mathrm{A}_{\mathrm{A}}$ for all values $(n=145)$. (a) N-HDL $\mathrm{A}_{\mathrm{A}}$ with C-LDL $\left(r^{2}=0.89\right)$. (b) N-HDL with D-LDL $\left(r^{2}=0.80\right)$. (c) D-LDL with C-LDL $\left(r^{2}=0.86\right)$.

of patients, as compared to $36 \%$ between C-LDL and NHDL and 34\% between C-LDL and D-LDL. This difference approached statistical significance when comparing DLDL and N-HDL to C-LDL $(P=0.064)$. Furthermore, when analyzed from a numerical perspective of clinical equivalence, values within 5 or $10 \mathrm{mg} / \mathrm{dL}$ of each other, this was least commonly observed with $\mathrm{N}-\mathrm{HDL}_{\mathrm{A}}$ and $\mathrm{D}$-LDL in the baseline and entire cohort (Figure 2). A statistically significant difference was observed between $\mathrm{N}-\mathrm{HDL}_{\mathrm{A}}$ versus
D-LDL and C-LDL versus D-LDL for both 5 and $10 \mathrm{mg} / \mathrm{dL}$ for the entire cohort (Figure 2(b)).

Clinical discordance (as defined in Section 2) between non-HDL and the 2 measures of LDL was noted on at least 1 occasion in $47 \%$ of patients with respect to D-LDL and in $37 \%$ with respect to C-LDL, while C-LDL and D-LDL were discordant in only $30 \%$ of patients $(P=0.075)$. A similar pattern emerged when looking at only the baseline lipid values, with discordance noted between N-HDL and 


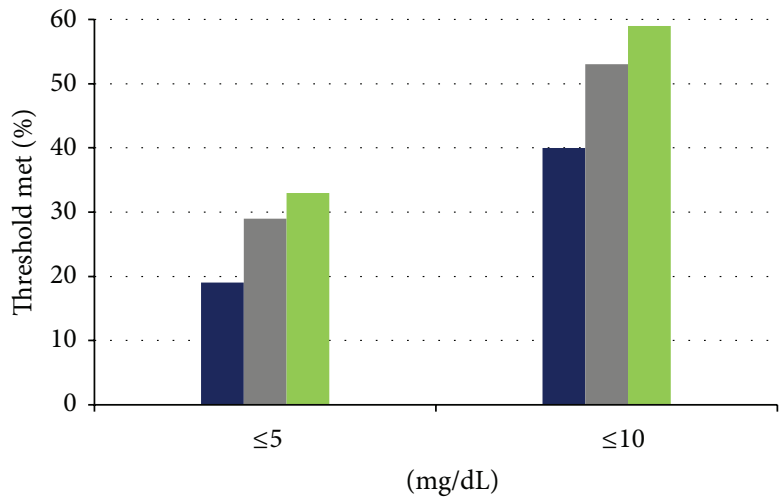

- N-HDL $\mathrm{A}_{\mathrm{A}}$ versus D-LDL

- N-HDL $L_{\mathrm{A}}$ versus C-LDL

- C-LDL versus D-LDL

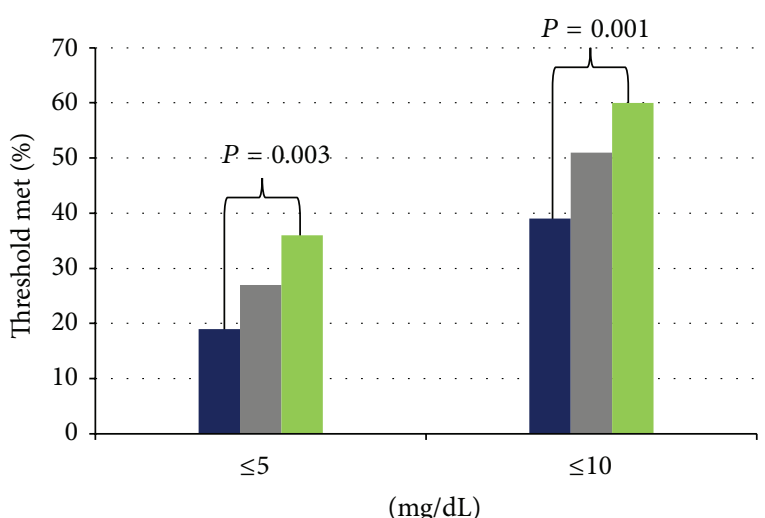

- N-HDL $\mathrm{A}_{\mathrm{A}}$ versus D-LDL

- N-HDL $L_{A}$ versus C-LDL

- C-LDL versus D-LDL

(b)

Figure 2: Percentage of patients whose values are near each other from a numerical perspective, $<5$ and $<10 \mathrm{mg} / \mathrm{dL}$. (a) Baseline values $(n=80)$, approached statistical significance for the between group differences $(P=0.054)$. (b) All values in the study $(n=145)$.

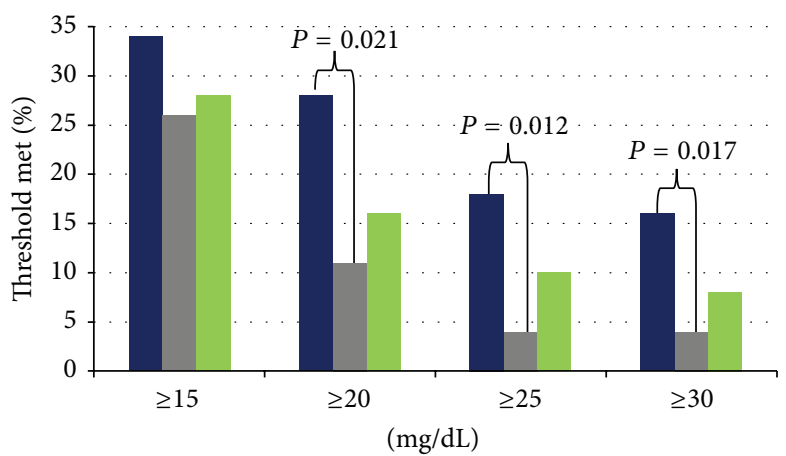

- N-HDL $\mathrm{A}_{\mathrm{A}}$ versus D-LDL

- N-HDL $\mathrm{A}_{\mathrm{A}}$ versus C-LDL

- C-LDL versus D-LDL

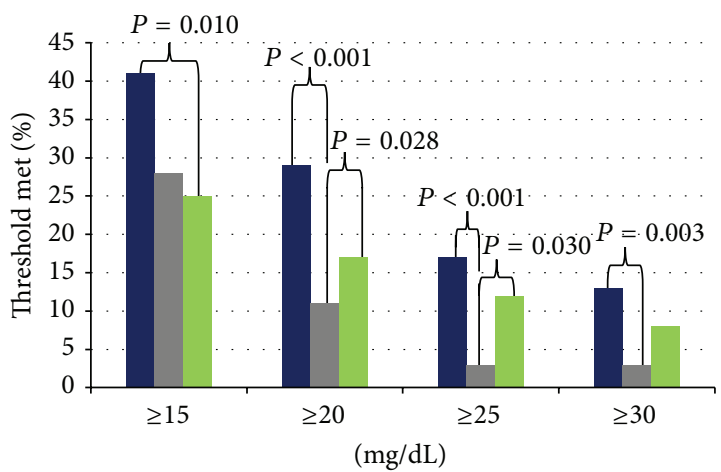

- $\mathrm{N}-\mathrm{HDL}_{\mathrm{A}}$ versus D-LDL

- N-HDL ${ }_{\mathrm{A}}$ versus C-LDL

- C-LDL versus D-LDL

(b)

Figure 3: Percentage of patients with a difference between $\mathrm{N}-H D L_{\mathrm{A}}$, D-LDL and C-LDL of 15, 20, 25, and $30 \mathrm{mg} / \mathrm{dL}$. (a) Baseline values $(n=80)$. (b) All values in the study $(n=145)$.

D-LDL in $31 \%$ of patients, as compared to $23 \%$ between $\mathrm{N}$ HDL and C-LDL and 15\% between direct and calculated LDL $(P=0.039$ versus $\mathrm{N}-\mathrm{HDL}$ and $\mathrm{D}-\mathrm{LDL})$.

Moreover, a clinically meaningful percentage of patients had a greater than $20 \mathrm{mg} / \mathrm{dL}$ difference between the various measures across the dataset, for both baseline and all values (Figure 3). This was statistically significantly more common when analyzing all values, between $\mathrm{N}-\mathrm{HDL}_{\mathrm{A}}$ and $\mathrm{D}-\mathrm{LDL}$, $29 \%$, when compared to C-LDL and $\mathrm{N}-\mathrm{HDL}_{\mathrm{A}}, 11 \%(P<$ $0.001)$ or C-LDL and D-LDL, 17\% $(P=0.028)$ (Figure 3(b)). Similar statistically significant and clinically meaningful findings were observed when analyzing only the baseline values (Figure 3(a)). A number of patients manifested even larger differences between the lipid measures, differences that clinicians would consider highly meaningful. This was observed most frequently when comparing $\mathrm{N}-\mathrm{HDL}_{\mathrm{A}}$ and $\mathrm{D}$ LDL, and was statistically significant for a number of values and lipid comparisons (Figure 3).

Similarly, the mean absolute difference between the various lipid measures was the greatest between D-LDL and $\mathrm{N}-\mathrm{HDL}_{\mathrm{A}}$, approximately $16 \mathrm{mg} / \mathrm{dL}$, as compared to approximately $11 \mathrm{mg} / \mathrm{dL}$ for N-HDL $\mathrm{A}_{\mathrm{A}}$ to C-LDL and D-LDL to C-LDL (Figure 4).

\section{Discussion}

Our analysis demonstrates a linear correlation between "adjusted" N-HDL and measures of LDL used in clinical practice, calculated and direct LDL. Despite this correlation, clinical discordance was observed in more than one in 


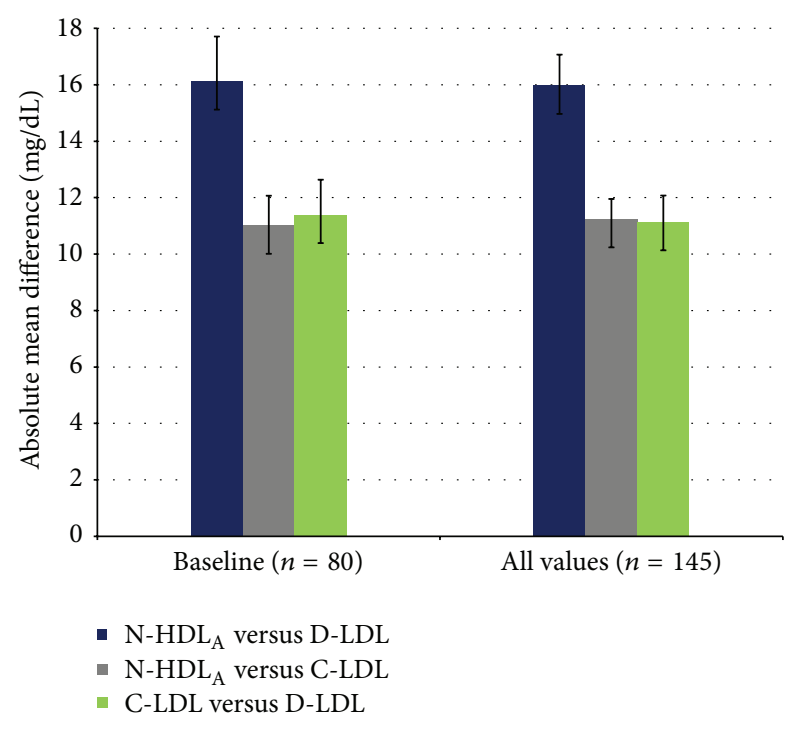

FIgURE 4: Mean absolute difference with standard deviation between N-HDL ${ }_{\mathrm{A}}, \mathrm{D}$-LDL, and C-LDL for baseline values $(n=80)$ and all values $(n=145)$ in the study. The difference and standard deviation are the largest between $\mathrm{N}-\mathrm{HDL}_{\mathrm{A}}$ and $\mathrm{D}-\mathrm{LDL}$ in both cohorts. Between-group differences were statistically significant, with $\mathrm{N}-\mathrm{HDL}_{\mathrm{A}}$ and D-LDL significantly different from both of the other lipid measurements for the baseline and entire cohorts, $P=$ 0.010 and $<0.001$, respectively.

three patients, and it was more common for N-HDL to be discordant than concordant with either C-LDL or DLDL. It is evident that N-HDL does not consistently provide similar clinical information to either measures of LDL in individual patients, a finding parallel to what we had seen previously when comparing C-LDL to D-LDL. This dissociation between clinical concordance and correlation results from the correlation coefficient being a summary statistic, rather than a statistic that defines relationships in individual patients.

Moreover, N-HDL and D-LDL, the recommended alternative lipid measures when C-LDL is inaccurate $[1,3]$ appear to be the most divergent of the three lipid "pairs," with $\mathrm{N}-\mathrm{HDL}_{\mathrm{A}}$ generally lowest, D-LDL highest, and C-LDL intermediate. Similarly, the average difference between lipid "pairs" was the greatest between $\mathrm{N}-\mathrm{HDL}_{\mathrm{A}}$ and D-LDL. N$\mathrm{HDL}_{\mathrm{A}}$ and D-LDL had the fewest number of patients whose values were very close to each other, within $5 \mathrm{mg} / \mathrm{dL}$, and the greatest number with highly discrepant values (e.g., $>20 \mathrm{mg} / \mathrm{dL}$ ). Furthermore, $\mathrm{N}-\mathrm{HDL}_{\mathrm{A}}$ and $\mathrm{D}-\mathrm{LDL}$ had the fewest concordant and most discordant values. Consistent with these findings, the weakest correlation was between $\mathrm{N}$ HDL and D-LDL.

Consequently, when C-LDL is inaccurate, recommending measurement of either N-HDL or D-LDL appears questionable, as these 2 lipid measures may not reliably provide the same clinical information in individual patients. Adopting such an approach will result in different treatment decisions, resulting in less intensive therapy when the primary target is $\mathrm{N}-\mathrm{HDL}$, and more intensive therapy, even with respect to $\mathrm{C}$-LDL, when D-LDL is the primary target. As there are fewer extreme differences from a numerical perspective between N-HDL and C-LDL, and N-HDL affords the additional advantages of the absence of additional cost and potential superiority to even C-LDL in predicting cardiovascular risk [6-11], the use of N-HDL seems preferable in situations where $\mathrm{C}-\mathrm{LDL}$ is inaccurate. The use of non-HDL in this setting is consistent with the growing body of support for non-HDL as a target of therapy [6-11], with some even advocating that it replaces calculated LDL as the primary target of therapy [12].

4.1. Study Limitations. Our study is limited by the use of a single commercial assay, in a modest number of a select, male, veteran population from a single center. Criteria for clinical concordance and discordance were based on our understanding of what clinicians would consider clinically meaningful. In addition, our patients are not representative of those in whom assessment of D-LDL is recommended, as all were fasting and had triglyceride levels of less than $400 \mathrm{mg} / \mathrm{dL}$. Despite these limitations, our study remains relevant for a number of reasons. Clinicians routinely use commercially available D-LDL assays, while our criteria for clinical concordance and discordance consisted of 3 criteria, any 1 of which clinicians may consider clinically meaningful. Moreover, N-HDL becomes more valuable as a predictor of risk as the triglycerides increase and non-HDL-C represents a secondary target of therapy when triglycerides are greater than $200 \mathrm{mg} / \mathrm{dL}$ [1].

\section{Conclusion}

N-HDL and D-LDL are the most divergent of the various lipid "pairs", on opposite "poles" of C-LDL, and thus do not appear to be clinically equivalent. The current findings strengthen our previous recommendation that N-HDL, and not D-LDL, be used in clinical situations where C-LDL is inaccurate, as $\mathrm{N}-\mathrm{HDL}$ is a validated target of therapy, free of additional cost, and incorporated into existing guidelines. The clinical discordance between all 3 lipid measures, N-HDL, D-LDL, and C-LDL, raises the issue of whether any of these lipid measures is the best predictor of cardiovascular risk and begs the question of whether alternative lipid measures, such as apoB or LDL particle number, should become the primary target of therapy [13-16]. Prospective research is needed to compare all these lipid measures to identify the best predictor of cardiovascular risk.

\section{Conflict of Interests}

There are no conflict of interests to report on the part of any of the authors.

\section{References}

[1] J. I. Cleeman, "Executive summary of the third report of the National Cholesterol Education Program (NCEP) expert panel on detection, evaluation, and treatment of high blood cholesterol in adults (adult treatment panel III)," Journal of the American Medical Association, vol. 285, no. 19, pp. 2486-2497, 2001. 
[2] R. McPherson, J. Frohlich, G. Fodor, and J. Genest, "Canadian Cardiovascular Society position statement-recommendations for the diagnosis and treatment of dyslipidemia and prevention of cardiovascular disease," Canadian Journal of Cardiology, vol. 22, no. 11, pp. 913-927, 2006.

[3] S. M. Grundy, J. I. Cleeman, C. N. Merz et al., "National Heart, Lung, and Blood Institute, American College of Cardiology Foundation, American Heart Association. Implications of recent clinical trials for the National Cholesterol Education Program Adult Treatment Panel III guidelines," Circulation, vol. 110, pp. 227-239, 2004.

[4] L. Baruch, S. Agarwal, B. Gupta et al., "Is directly measured low-density lipoprotein clinically equivalent to calculated lowdensity lipoprotein?" Journal of clinical lipidology, vol. 4, no. 4, pp. 259-264, 2010.

[5] K. A. Foley, R. J. Simpson, J. R. Crouse, T. W. Weiss, L. E. Markson, and C. M. Alexander, "Effectiveness of statin titration on low-density lipoprotein cholesterol goal attainment in patients at high risk of atherogenic events," American Journal of Cardiology, vol. 92, no. 1, pp. 79-81, 2003.

[6] J. J. Kastelein, W. A. van der Steeg, I. Holme et al., "Lipids, apolipoproteins, and their ratios in relation to cardiovascular events with statin treatment," Circulation, vol. 117, pp. 30023009, 2008.

[7] P. M. Ridker, N. Rifai, N. R. Cook, G. Bradwin, and J. E. Buring, "Non-HDL cholesterol, apolipoproteins A-I and B100, standard lipid measures, lipid ratios, and CRP as risk factors for cardiovascular disease in women," Journal of the American Medical Association, vol. 294, no. 3, pp. 326-333, 2005.

[8] T. Pischon, C. J. Girman, F. M. Sacks, N. Rifai, M. J. Stampfer, and E. B. Rimm, "Non-high-density lipoprotein cholesterol and apolipoprotein B in the prediction of coronary heart disease in men," Circulation, vol. 112, no. 22, pp. 3375-3383, 2005.

[9] Y. Cui, R. S. Blumenthal, J. A. Flaws et al., "Non-high-density lipoprotein cholesterol level as a predictor of cardiovascular disease mortality," Archives of Internal Medicine, vol. 161, pp. 1413-1419, 2001.

[10] B. J. Arsenault, J. S. Rana, E. S. G. Stroes et al., "Beyond low-density lipoprotein cholesterol. Respective contributions of non-high-density lipoprotein cholesterol levels, triglycerides, and the total cholesterol/high-density lipoprotein cholesterol ratio to coronary heart disease risk in apparently healthy men and women," Journal of the American College of Cardiology, vol. 55, no. 1, pp. 35-41, 2009.

[11] J. G. Robinson, S. Wang, B. J. Smith, and T. A. Jacobson, "Meta-analysis of the relationship between non-high-density lipoprotein cholesterol reduction and coronary heart disease risk," Journal of the American College of Cardiology, vol. 53, no. 4, pp. 316-322, 2009.

[12] M. J. Blaha, R. S. Blumenthal, E. A. Brinton, and T. A. Jacobson, "The importance of non-HDL cholesterol reporting in lipid management," Journal of Clinical Lipidology, vol. 2, no. 4, pp. 267-273, 2008.

[13] J. D. Brunzell, M. Davidson, C. D. Furberg et al., "Lipoprotein management in patients with cardiometabolic risk. Consensus conference report from the american diabetes association and the american college of cardiology foundation," Journal of the American College of Cardiology, vol. 51, no. 15, pp. 1512-1524, 2008.

[14] J. H. Contois, J. P. McConnell, A. A. Sethi et al., "Apolipoprotein $B$ and cardiovascular disease risk: position statement from the AACC lipoproteins and vascular diseases division working group on best practices," Clinical Chemistry, vol. 55, no. 3, pp. 407-419, 2009.

[15] M. H. Davidson, C. M. Ballantyne, T. A. Jacobson et al., "Clinical utility of inflammatory markers and advanced lipoprotein testing: advice from an expert panel of lipid specialists," Journal of Clinical Lipidology, vol. 5, pp. 338-567, 2011.

[16] S. M. Boekholdt, B. J. Arsenault, S. Mora et al., "Association of LDL cholesterol, non-HDL cholesterol, and apolipoprotein B levels with risk of cardiovascular events among patients treated with statins: a meta-analysis," The Journal of the American Medical Association, vol. 307, pp. 1302-1309, 2012. 


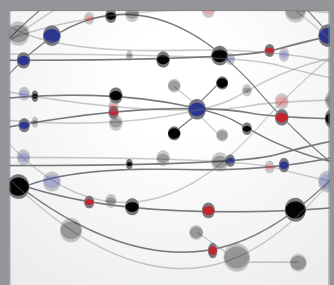

The Scientific World Journal
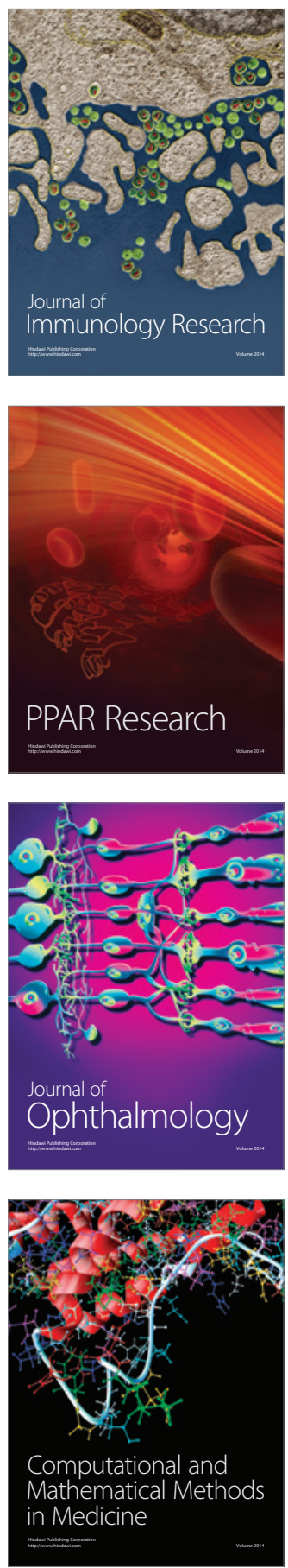

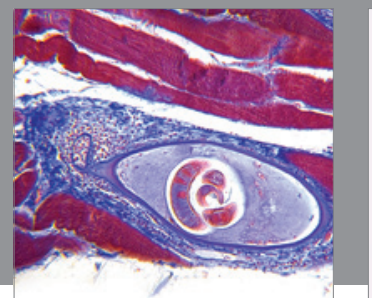

Gastroenterology

Research and Practice
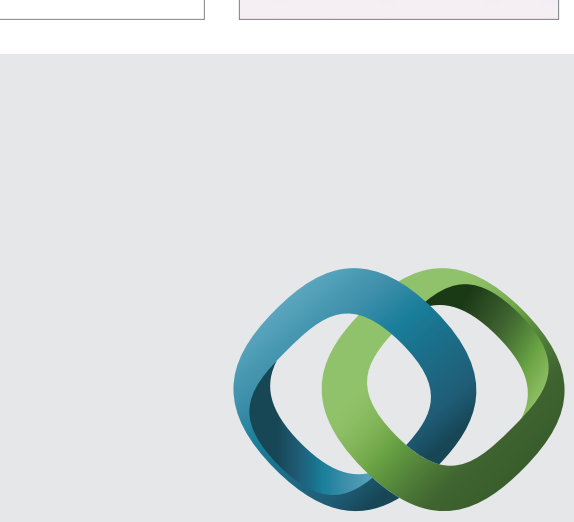

\section{Hindawi}

Submit your manuscripts at

http://www.hindawi.com
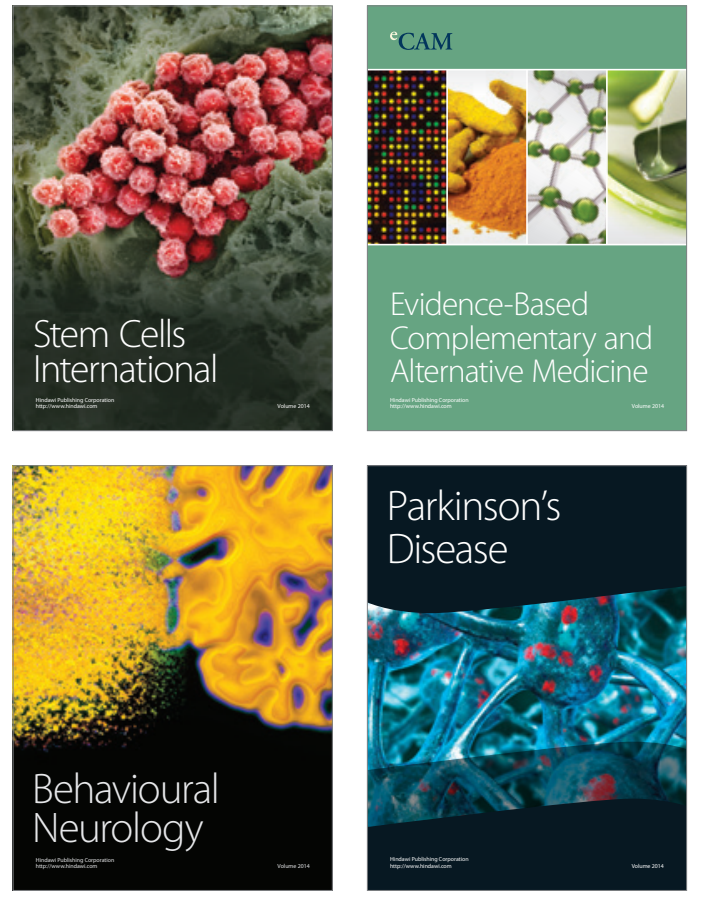
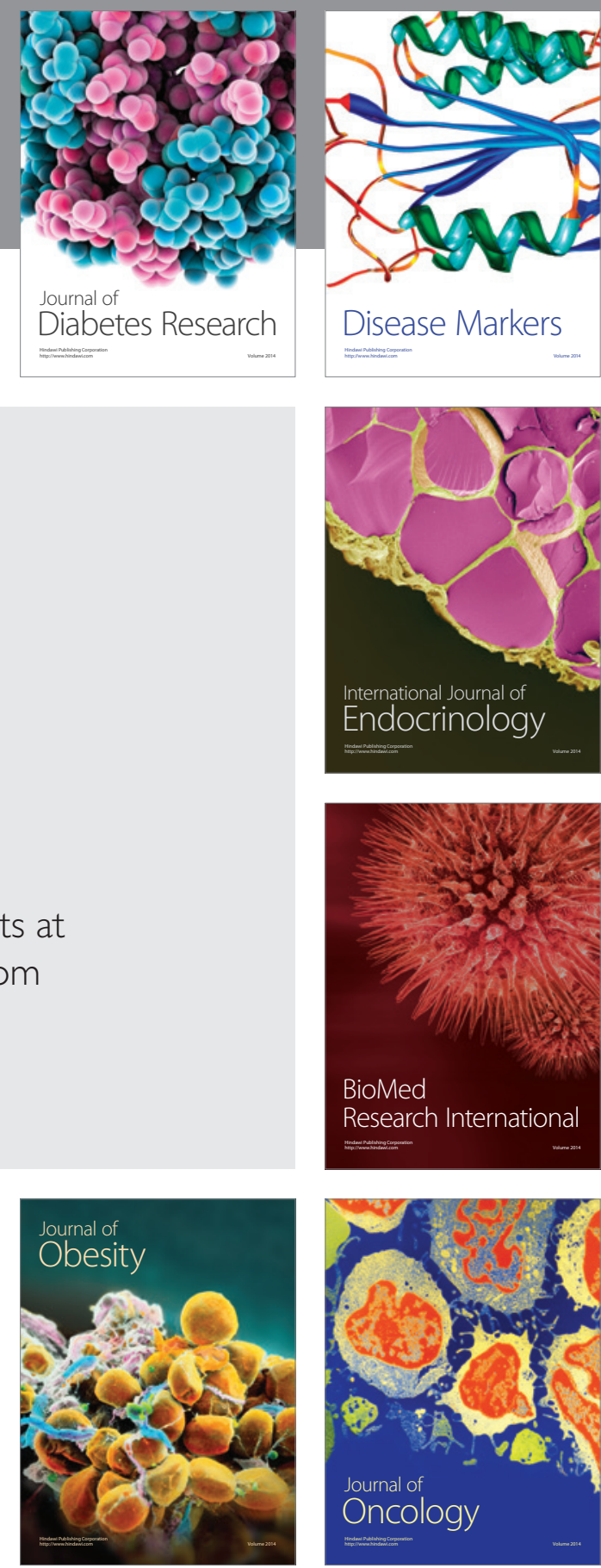

Disease Markers
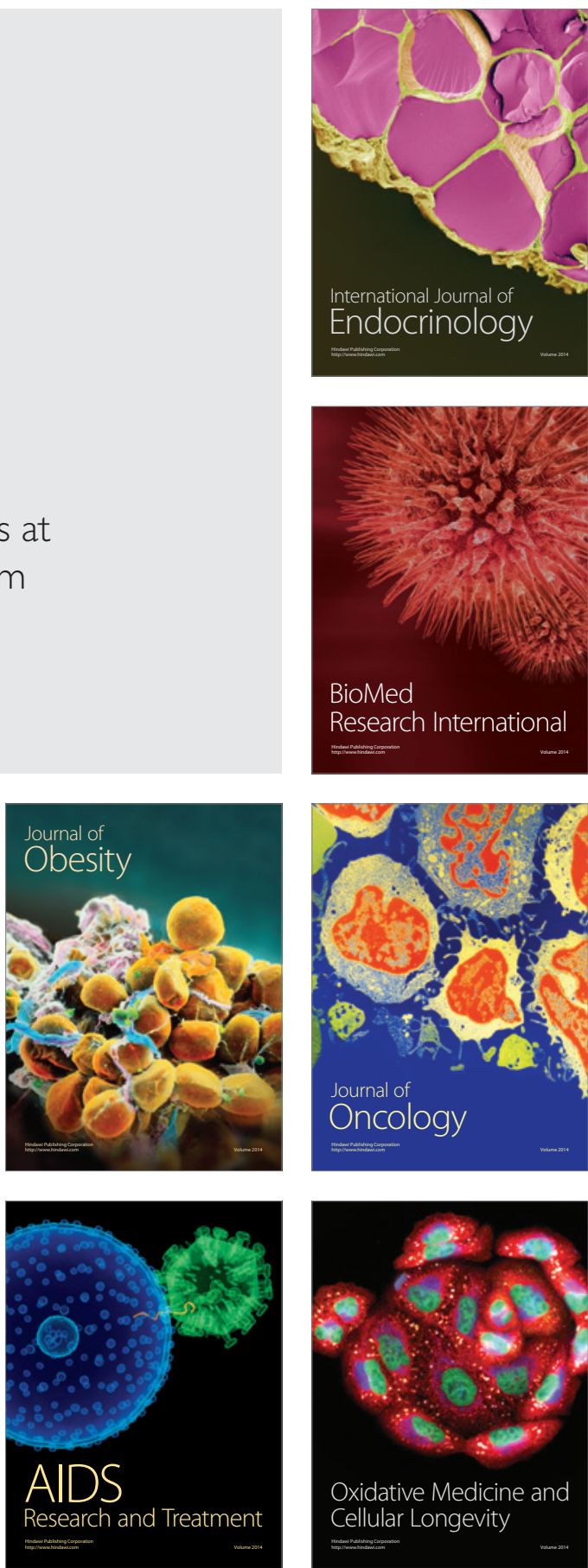